\title{
Automatic Information and Safety Systems for Driving Assistance
}

\author{
Miguel Oliveira \\ Institute of Electronics and Telematics Engineering of Aveiro, Campus Universitario de Santiago, 3800 Aveiro, Portugal \\ Advisors: Vitor Santos, Angel D. Sappa \\ Date and location of PhD thesis defense: 21 February 2013, University of Aveiro
}

Received 17th Feb 2014; accepted 27th May 2014

\section{Abstract}

The main object of this thesis is the study of algorithms for automatic information processing and representation, in particular information provided by onboard sensors ( $2 \mathrm{D}$ and $3 \mathrm{D})$, to be used in the context of driving assistance. The work focuses on some of the problems facing todays Autonomous Driving (AD) systems and Advanced Drivers Assistance Systems (ADAS). The document is composed of two parts. The first part describes the design, construction and development of three robotic prototypes, including remarks about onboard sensors, algorithms and software architectures. These robots were used as test beds for testing and validating the developed techniques; additionally, they have participated in several autonomous driving competitions with very good results. The second part presents several algorithms for generating intermediate representations of the raw sensor data. They can be used to enhance existing pattern recognition, detection or navigation techniques, and may thus benefit future AD or ADAS applications. Since vehicles often contain a large amount of sensors of different natures, intermediate representations are particularly advantageous; they can be used for tackling problems related with the diverse nature of the data $(2 \mathrm{D}, 3 \mathrm{D}$, photometric, etc.), with the asynchrony of the data (multiple sensors streaming data at different frequencies), or with the alignment of the data (calibration issues, different sensors providing different measurements of the same object). Within this scope, novel techniques are proposed for computing a multi-camera multi-modal inverse perspective mapping representation, executing color correction between images for obtaining quality mosaics, or to produce a scene representation based on polygonal primitives that can cope with very large amounts of 3D and 2D data, including the ability of refining the representation as new information is continuously received.

Thus, the main focus is on the development of algorithms and techniques for enhanced representation of onboard sensor data. Finding alternative sensor data representations is very useful, since it may improve the effectiveness of algorithms used in the perception of road-like scenarios. Additionally, these intermediate scene representations may be advantageous since they are devised to cope with the large amounts of data, thus enabling real-time computation. One example is the Velodyne laser, which is now considered a standard in autonomous vehicles, but that generates 1.3 million range data points per second. It was shown that classical approaches (such as 3D triangulations) are unable to process such large amounts of data in real-time. A new

Correspondence to: mriem@ua.pt

Recommended for acceptance by Alicia Fornés and Volkmar Frinken ELCVIA ISSN:1577-5097

Published by Computer Vision Center / Universitat Autònoma de Barcelona, Barcelona, Spain 
approach is proposed which is six times faster than alternative methodologies. Another advantage of intermediate representations is that they can cope with the asynchrony of the data in multi sensorial setups. This is particularly important, since that autonomous vehicles are equipped with a vast number of sensors of varied nature. In detail, the following contributions were made:

- A multi-camera multi-modal Inverse Perspective Mapping methodology that uses data from a Laser Range Finder to enhance the IPM resulting image. Results have shown that the images produced by this approach are more precise than images computed using the classical IPM approaches [1];

- Three alternative color correction algorithms for obtaining quality mosaics. Results have shown that these techniques are more efficient and robust than the state of the art $[2,3,4]$;

- A methodology based on geometric polygonal primitives, that computes an accurate geometric scene representation in approximately one sixth of the time of the fastest 3D triangulation approach tested. Additionally, a method was proposed to refine the polygonal primitive based representation over time;

- A methodology for texture mapping the geometric polygonal primitive representation using Data Dependent Triangulation. Additionally, a methodology for refining the models texture over time was proposed;

- The integration of some of the algorithms described in previous lines into three robotic prototypes. Testing and evaluation of the algorithms in real platforms used in several competitions $[5,6,7]$.

\section{References}

[1] M. Oliveira and V. Santos. Multi-camera active perception system with variable image perspective for mobile robot navigation. Intl. Conf. on Autonomous Robot Systems and Competitions, Aveiro, 2008. Best conference paper award.

[2] M. Oliveira, A. D. Sappa, and V. Santos. Unsupervised local color transfer for coarsely registered images. IEEE Proc. of Intl. Conf. on Computer Vision and Pattern Recognition, Colorado Springs, 201-208, 2011.

[3] M. Oliveira, A. D. Sappa, and V. Santos. Color correction using 3d Gaussian mixture models. Proc. of Intl. Conf. on Image Analysis and Recognition, Aveiro, 97-106, 2012.

[4] M. Oliveira, A. D. Sappa, and V. Santos. Color correction for onboard multi-camera systems using 3d Gaussian mixture models. Proc. of Intelligent Vehicles Symposium, Madrid, 299-303, 2012.

[5] M. Oliveira, P. Stein, J. Almeida, and V. Santos. Modular scalable architecture for the navigation of the atlas autonomous robots. Proc. of Intl. Conf. on Autonomous Robot Systems and Competitions, Castelo Branco, 2009.

[6] M. Oliveira and V. Santos. Autonomous driving competition: Perception approaches used in the atlas project. Proc. of Intl. Conf. on Autonomous Robot Systems and Competitions, Lisboa, 2011.

[7] V. Santos, J. Almeida, E. Avila, D. Gameiro, M. Oliveira, R. Pascoal, R. Sabino, and P. Stein. Atlascar technologies for a computer assisted driving system on board a common automobile. IEEE Proc. of Intl. Conf. on Intelligent Transportation Systems, Madeira, 1421-1427, 2010. 